\title{
Low-frequency incommensurate magnetic response in strongly correlated systems
}

\author{
A. Sherman ${ }^{1}$ and M. Schreiber ${ }^{2}$ \\ ${ }^{1}$ Institute of Physics, University of Tartu, Riia 142, 51014 Tartu, Estonia \\ ${ }^{2}$ Institut für Physik, Technische Universität, D-09107 Chemnitz, Federal Republic of Germany
}

(Dated: October 31, 2018)

\begin{abstract}
It is shown that in the $t-J$ model of $\mathrm{Cu}-\mathrm{O}$ planes at low frequencies the dynamic spin structure factor is peaked at incommensurate wave vectors $\left(\frac{1}{2} \pm \delta, \frac{1}{2}\right),\left(\frac{1}{2}, \frac{1}{2} \pm \delta\right)$. The incommensurability is connected with the momentum dependencies of the magnon frequency and damping near the antiferromagnetic wave vector. The behavior of the incommensurate peaks is similar to that observed in $\mathrm{La}_{2-x}(\mathrm{Ba}, \mathrm{Sr})_{x} \mathrm{CuO}_{4+y}$ and $\mathrm{YBa}_{2} \mathrm{Cu}_{3} \mathrm{O}_{7-y}$ : for hole concentrations $0.02<x \lesssim 0.12$ we find that $\delta$ is nearly proportional to $x$, while for $x>0.12$ it tends to saturation. The incommensurability disappears with increasing temperature. Generally the incommensurate magnetic response is not accompanied by an inhomogeneity of the carrier density.
\end{abstract}

PACS numbers: $71.10 . \mathrm{Fd}, 74.25 . \mathrm{Ha}$

One of the most interesting features of the inelastic neutron scattering in lanthanum cuprates is that for hole concentrations $x \lesssim 0.04$ the spin fluctuation scattering remains commensurate: it is peaked at the antiferromagnetic wave vector $\mathbf{Q}=\left(\frac{1}{2}, \frac{1}{2}\right)$ in the reciprocal lattice units $2 \pi / a$ with the lattice period $a$. However, for larger $x$ and low energy transfers the spin fluctuations become incommensurate with scattering peaks shifted to the four symmetric positions $\left(\frac{1}{2} \pm \delta, \frac{1}{2}\right)$ and $\left(\frac{1}{2}, \frac{1}{2} \pm \delta\right)$ [1]. For $x \lesssim 0.12$ the incommensurability parameter $\delta$ is approximately equal to $x$ and varies nearly linearly with the temperature of the superconducting transition $T_{c}$ [2]. For larger $x$ the parameter saturates near the value $\delta \approx 0.12$. The incommensurate response was observed both below and above $T_{c}$, 3]. There are no clear experimental indications that in $\mathrm{La}_{2-x} \mathrm{Sr}_{x} \mathrm{CuO}_{4}$ the dynamic magnetic incommensurability is connected with lattice distortions. However, for $x \approx 0.12$ when the static incommensurability is observed for temperatures $T \lesssim T_{c}$ a remarkable softening of longitudinal sound waves and soft optical phonons were observed 4]. This softening is a manifestation of the incipient structural transition from the low-temperature orthorhombic to the low-temperature tetragonal phase and the appearance of the static incommensurability is connected with the pinning of the dynamic incommensurate spin correlations by this lattice instability [4].

The experimental data suggest that the magnetic incommensurability emerges due to doped holes. Several approaches have been used to clarify the underlying mechanism. As possible candidates the Fermi surface nesting [5], mean-field spiral [6] and stripe 7] phases, and phenomenological marginal Fermi liquid [8] were considered. In works based on the Fermi surface nesting the difference in the low-frequency magnetic response in $\mathrm{La}_{2-x} \mathrm{Sr}_{x} \mathrm{CuO}_{4}$ and $\mathrm{YBa}_{2} \mathrm{Cu}_{3} \mathrm{O}_{7-y}$ was attributed to the difference in their Fermi surfaces calculated in the meanfield approximation. However, recently the incommensurability which is analogous to that observed in lan- thanum cuprates was also detected in $\mathrm{YBa}_{2} \mathrm{Cu}_{3} \mathrm{O}_{7-y}[\underline{9}]$. This result indicates that the low-frequency incommensurate magnetic response may be a common property of cuprates. This is especially interesting because the overall magnetic response of these two classes of crystals differs essentially. As known, in $\mathrm{YBa}_{2} \mathrm{Cu}_{3} \mathrm{O}_{7-y}$ and some other high $T_{c}$ cuprates the resonance peak is observed [10] at somewhat larger frequencies $\omega=20-40 \mathrm{meV}$ than the frequencies of incommensurate response, while no such peak was detected in lanthanum cuprates [1]. In works based on the stripe mechanism the magnetic incommensurability is connected with the appearance of the charge density wave. In neutron scattering such a wave is observed only in the low-temperature tetragonal or the low-temperature less-orthorhombic phases where the wave is stabilized by the corrugated pattern of the in-plane lattice potential [12]. Among lanthanum cuprates $\mathrm{La}_{2-x} \mathrm{Ba}_{x} \mathrm{CuO}_{4}$ and $\mathrm{La}_{2-y-x} \mathrm{Nd}_{y} \mathrm{Sr}_{x} \mathrm{CuO}_{4}$ have these phases. In $\mathrm{La}_{2-x} \mathrm{Sr}_{x} \mathrm{CuO}_{4}$ in the low-temperature orthorhombic phase no indications of the charge density wave were observed in neutron scattering [13].

In this letter we demonstrate that the low-frequency incommensurate response can be interpreted based on the $t-J$ model of $\mathrm{Cu}-\mathrm{O}$ planes. We use the general form of the spin spectral function $B(\mathbf{k} \omega)$ obtained [14] in Mori's projection operator technique. This approach allows us to take proper account of strong electron correlations inherent in cuprates. In calculating the magnon damping the known shape of the spin-polaron band [15] which forms the Fermi surface at low $x$ was used. The mechanism of the appearance of the incommensurability is the following: at low frequencies $B(\mathbf{k} \omega)$ is reduced to the quotient of the magnon damping $\Gamma(\mathbf{k})$ and the fourth power of the magnon frequency $\omega_{\mathbf{k}}$. Near $\mathbf{Q}$ this frequency is an increasing function of $|\mathbf{k}-\mathbf{Q}|$. Thus, $B(\mathbf{k} \omega)$ will be peaked at incommensurate momenta if $\Gamma(\mathbf{k})$ is also an increasing function of $|\mathbf{k}-\mathbf{Q}|$. This takes place indeed because the magnon damping connected with the decay into an electron-hole pair is approximately propor- 
tional to $\mathcal{S}=\sum_{\mathbf{k}^{\prime}}\left[1-n_{F}\left(\varepsilon_{\mathbf{k}+\mathbf{k}^{\prime}}-\mu\right)\right] n_{F}\left(\varepsilon_{\mathbf{k}^{\prime}}-\mu\right)$ where $n_{F}(\omega)=[\exp (\omega / T)+1]^{-1}, \varepsilon_{\mathbf{k}}$ is the hole dispersion and $\mu$ the chemical potential. Due to the approximate periodicity of $\varepsilon_{\mathbf{k}}$ with the period $\mathbf{Q}$ (connected with the short-range antiferromagnetic order) $\mathcal{S}=0$ for $\mathbf{k}=\mathbf{Q}$ and grows with growing $|\mathbf{k}-\mathbf{Q}|$.

The spin spectral function, connected with the retarded spin Green's function $D(\mathbf{k} \omega)=\left\langle\left\langle s_{\mathbf{L}}^{z} \mid s_{-\mathbf{k}}^{z}\right\rangle\right\rangle$ by the relation $B(\mathbf{k} \omega)=-\pi^{-1} \operatorname{Im} D(\mathbf{k} \omega)$, reads [14]

$$
B(\mathbf{k} \omega)=-\frac{\pi^{-1} \omega \Gamma(\mathbf{k} \omega)}{\left[\omega^{2}-f^{-1} \omega \Theta(\mathbf{k} \omega)-\omega_{\mathbf{k}}^{2}\right]^{2}+f^{-2} \omega^{2} \Gamma^{2}(\mathbf{k} \omega)},
$$

where $s_{\mathbf{k}}^{z}$ is the $z$-component of the spin- $\frac{1}{2}$ operator describing localized $\mathrm{Cu}$ spins, $f=4 J C_{1}\left(\gamma_{\mathbf{k}}-1\right)$ with $\gamma_{\mathbf{k}}=\frac{1}{4} \sum_{\mathbf{a}} \exp (i \mathbf{k a})$, a are four vectors connecting nearest neighbor sites in the square lattice of the $2 \mathrm{D} t-J$ model, $J$ is its exchange parameter, $C_{1}=2\left\langle s_{\mathbf{n}}^{z} s_{\mathbf{n}+\mathbf{a}}^{z}\right\rangle$ is the spin correlation on the neighbor sites $\mathbf{n}$ and $\mathbf{n}+\mathbf{a}$ with the statistical averaging denoted by the angular brackets. In the normal state the magnon frequency and the damping are given by the relations

$$
\begin{aligned}
\omega_{\mathbf{k}}^{2} & =16 \alpha J^{2}\left|C_{1}\right|\left(1-\gamma_{\mathbf{k}}\right)\left(\Delta+1+\gamma_{\mathbf{k}}\right), \\
\Gamma(\mathbf{k} \omega) & =\frac{32 \pi t^{2} J^{2}}{N^{2}} \frac{1-\exp (\omega / T)}{\omega} \\
& \times \sum_{\mathbf{k}_{1} \mathbf{k}_{2}} g_{\mathbf{k k}_{1} \mathbf{k}_{2}}^{2} \iint_{-\infty}^{\infty} d \omega_{1} d \omega_{2} n_{B}\left(\omega_{2}\right) \\
& \times\left[1-n_{F}\left(\omega_{1}\right)\right] n_{F}\left(\omega+\omega_{1}-\omega_{2}\right) B\left(\mathbf{k}_{2} \omega_{2}\right) \\
& \times A\left(\mathbf{k}_{1} \omega_{1}\right) A\left(\mathbf{k}+\mathbf{k}_{1}-\mathbf{k}_{2}, \omega+\omega_{1}-\omega_{2}\right),
\end{aligned}
$$

where $t$ is the hopping parameter of the $t$ - $J$ model, $N$ is the number of sites, $\alpha$ is the vertex parameter [16] which is of the order of unity, $g_{\mathbf{k k}_{1} \mathbf{k}_{2}}=\left(\gamma_{\mathbf{k}_{2}}+\frac{1}{4}\right)\left(\gamma_{\mathbf{k}_{2}-\mathbf{k}_{1}}-\right.$ $\left.\gamma_{\mathbf{k}_{1}}-\gamma_{\mathbf{k}+\mathbf{k}_{1}-\mathbf{k}_{2}}+\gamma_{\mathbf{k}+\mathbf{k}_{1}}\right), n_{B}(\omega)=[\exp (\omega / T)-1]^{-1}$, and $A(\mathbf{k} \omega)=-\pi^{-1} \operatorname{Im}\left\langle\left\langle a_{\mathbf{k} \sigma} \mid a_{\mathbf{k} \sigma}^{\dagger}\right\rangle\right\rangle$ is the hole spectral function with the operator $a_{\mathbf{k} \sigma}^{\dagger}$ creating a hole with the spin projection $\sigma= \pm 1$. In Eq. (2), the parameter $\Delta$ describes the spin gap at $\mathbf{Q}$ (see 16 and references therein). The correction $\Theta(\mathbf{k} \omega)$ to the magnon frequency can be obtained from $\Gamma(\mathbf{k} \omega)$ and the Kramers-Kronig relation. The dynamic structure factor measured in neutron scattering experiments is connected with $B(\mathbf{k} \omega)$ by the relation $S(\mathbf{k} \omega)=\pi[1-\exp (-\omega / T)]^{-1} B(\mathbf{k} \omega)$ [17.

If the magnons near $\mathbf{Q}$ are not overdamped, the dominant feature of the spectral function (11) is the maximum near $\omega=\omega_{\mathbf{Q}}=4 J\left(2 \alpha\left|C_{1}\right| \Delta\right)^{1 / 2}$. This maximum, the resonance peak, is observed in $\mathrm{YBa}_{2} \mathrm{Cu}_{3} \mathrm{O}_{7-y}$ and some other high $T_{c}$ cuprates [10]. Notice that its frequency $\omega=20-40 \mathrm{meV}$ is close to the frequency of the spin gap $\omega_{\mathbf{Q}}$ 14, 18]. In lanthanum cuprates the magnons near $\mathbf{Q}$ are apparently overdamped even in the underdoped region and due to the small superconducting gap the resonance peak was not observed in the superconducting

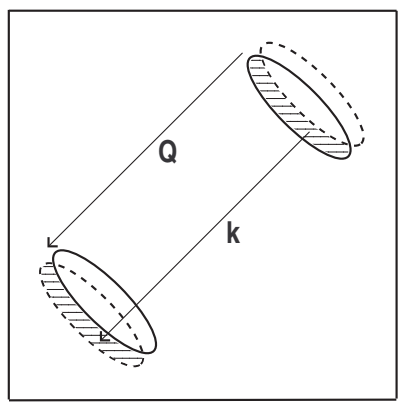

FIG. 1: The Brillouin zone of the square lattice. Solid curves are two of four ellipses forming the Fermi surface of the band (6) at small $x$. Dashed lines are the Fermi surface contours shifted by $\pm(\mathbf{k}-\mathbf{Q})$. Regions of $\mathbf{k}_{1}$ and $\mathbf{k}+\mathbf{k}_{1}$ contributing to the damping (7) are shaded.

state either [11, 18]. In this case the frequency dependence of the spectral function is determined by $\Gamma(\mathbf{k} \omega)$ rather than the resonance denominator in Eq. (11). In lanthanum cuprates the incommensurate response is observed for frequencies of the order of several millielectronvolts. For such frequencies Eq. (1) can be essentially simplified,

$$
B(\mathbf{k} \omega)=-\frac{\pi^{-1} \omega \Gamma(\mathbf{k} \omega)}{\omega_{\mathbf{k}}^{4}} .
$$

As seen from Eq. (2), $\omega_{\mathbf{k}} \approx\left[\omega_{\mathbf{Q}}^{2}+c^{2}(\mathbf{k}-\mathbf{Q})^{2}\right]^{1 / 2}$ near $\mathbf{Q}$ and therefore in Eq. (4) $\omega_{\mathbf{k}}^{-4}$ decreases rapidly with increasing $|\mathbf{k}-\mathbf{Q}|$. In this case for the function $B(\mathbf{k} \omega)$ to be peaked at incommensurate momenta near $\mathbf{Q}$ the numerator of the fraction (4), the magnon damping, has to be a growing function of $|\mathbf{k}-\mathbf{Q}|$.

To make sure that the damping may really have such a behavior let us consider the case of low hole concentrations when in Eq. (3) the hole and spin spectral functions can be approximated as [16]

$$
\begin{aligned}
A(\mathbf{k} \omega)= & \phi \delta\left(\omega-\varepsilon_{\mathbf{k}}+\mu\right), \\
B(\mathbf{k} \omega)= & \frac{1}{2} \sqrt{\frac{\left|C_{1}\right|}{\alpha}} \sqrt{\frac{1-\gamma_{\mathbf{k}}}{\Delta+1+\gamma_{\mathbf{k}}}} \\
& \times\left[\delta\left(\omega-\omega_{\mathbf{k}}\right)-\delta\left(\omega+\omega_{\mathbf{k}}\right)\right],
\end{aligned}
$$

where $\phi=\frac{1}{2}(1+x)$ and the dispersion of the spin-polaron band [15, 10]

$$
\begin{aligned}
\varepsilon_{\mathbf{k}} / t= & -2.3893+0.055358\left[\cos \left(2 k_{x}\right)+\cos \left(2 k_{y}\right)\right] \\
& +0.17857 \cos \left(k_{x}\right) \cos \left(k_{y}\right) .
\end{aligned}
$$

The approximate formula (6) reproduces the main known features of the spin-polaron band 15]. In particular, it has minima at $( \pm \pi / 2, \pm \pi / 2)$. The bottom of the band, $-2.5 t$, and its width, $0.4 t=2 J$, are set to be close to the values obtained in spin-wave and exact diagonalization calculations for $J=0.2 t$. This parameter ratio is close 


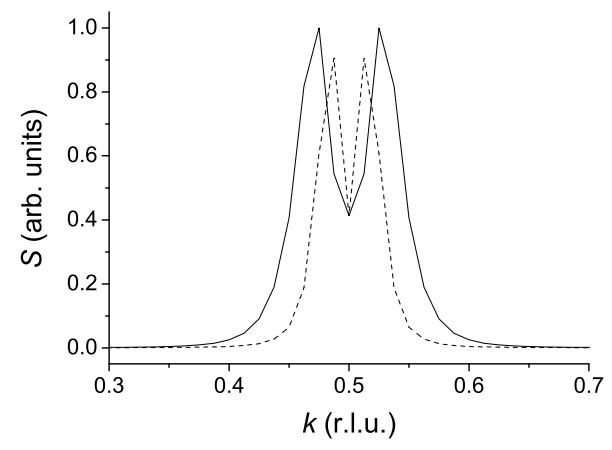

FIG. 2: The dynamic structure factor vs. wave vector along the edge (solid line) and the diagonal (dashed line) of the Brillouin zone for $x=0.06, T=0.01 t$, and $\omega=0.004 t$.

to that observed in hole-doped cuprates [19]. Also the shape of the band (6) and its Fermi surface are similar to those observed in oxychlorides and in $\mathrm{La}_{2}{ }_{x} \mathrm{Sr}_{x} \mathrm{CuO}_{4}$ at light and moderate doping [16, 20]. For low $x$ the Fermi surface of the band (6) consists of four ellipses centered at $( \pm \pi / 2, \pm \pi / 2)$. Two of them are shown in Fig. 1 Substituting Eq. (5) in Eq. (3) and taking into account that for $\mathbf{k} \approx \mathbf{Q}$ and small $\omega$ the region around $\mathbf{Q}$ makes the main contribution in the summation over $\mathbf{k}_{2}$ we find

$$
\begin{aligned}
\Gamma(\mathbf{k} \omega) & \approx \frac{32 \pi t^{2} J^{2} \phi^{2}}{N^{2}} \frac{1-\exp (\omega / T)}{\omega} \\
& \times \sum_{\mathbf{k}_{1}} g_{\mathbf{k k}_{1} \mathbf{Q}}^{2}\left[1-n_{F}\left(\varepsilon_{\mathbf{k}_{1}}-\mu\right)\right] n_{F}\left(\varepsilon_{\mathbf{k}+\mathbf{k}_{1}}-\mu\right) \\
& \times \sum_{\mathbf{k}_{2}} B\left(\mathbf{k}_{2}, \omega+\varepsilon_{\mathbf{k}_{1}}-\varepsilon_{\mathbf{k}+\mathbf{k}_{1}-\mathbf{k}_{2}}\right) \\
& \times n_{B}\left(\omega+\varepsilon_{\mathbf{k}_{1}}-\varepsilon_{\mathbf{k}+\mathbf{k}_{1}-\mathbf{k}_{2}}\right) .
\end{aligned}
$$

The dependence $\Gamma(\mathbf{k})$ is mainly determined by the sum $\mathcal{S}=\sum_{\mathbf{k}_{1}} g_{\mathbf{k k}_{1} \mathbf{Q}}^{2}\left[1-n_{F}\left(\varepsilon_{\mathbf{k}_{1}}-\mu\right)\right] n_{F}\left(\varepsilon_{\mathbf{k}+\mathbf{k}_{1}}-\mu\right)$. For low temperatures the regions of $\mathbf{k}_{1}$ and $\mathbf{k}+\mathbf{k}_{1}$ contributing to the sum are shaded in Fig. 1 The damping is roughly proportional to the area of these regions. Thus, for low $T$ and $x$ the damping grows with growing $|\mathbf{k}-\mathbf{Q}|$ which shifts the peaks in $B(\mathbf{k} \omega)$ to incommensurate positions.

Key elements for the validity of the above conclusion are the spin spectral function in Eq. (3) which is strongly peaked near $\mathbf{Q}$ and an approximate periodicity of the hole dispersion with the period $\mathbf{Q}$. Due to the doubling of the elementary cell in antiferromagnetically ordered crystals both these conditions have to be fulfilled in lightly and moderately doped cuprates which are characterized by the short-range antiferromagnetic ordering. A small damping of hole states is also necessary (the incommensurability was suppressed in the self-consistent calculations 16] due to a sizeable hole damping introduced for stabilizing the iteration procedure).

We used Eqs. (3)-(6) and parameters $\alpha, C_{1}$, and $\Delta$ obtained self-consistently [16] for calculating the dynamic
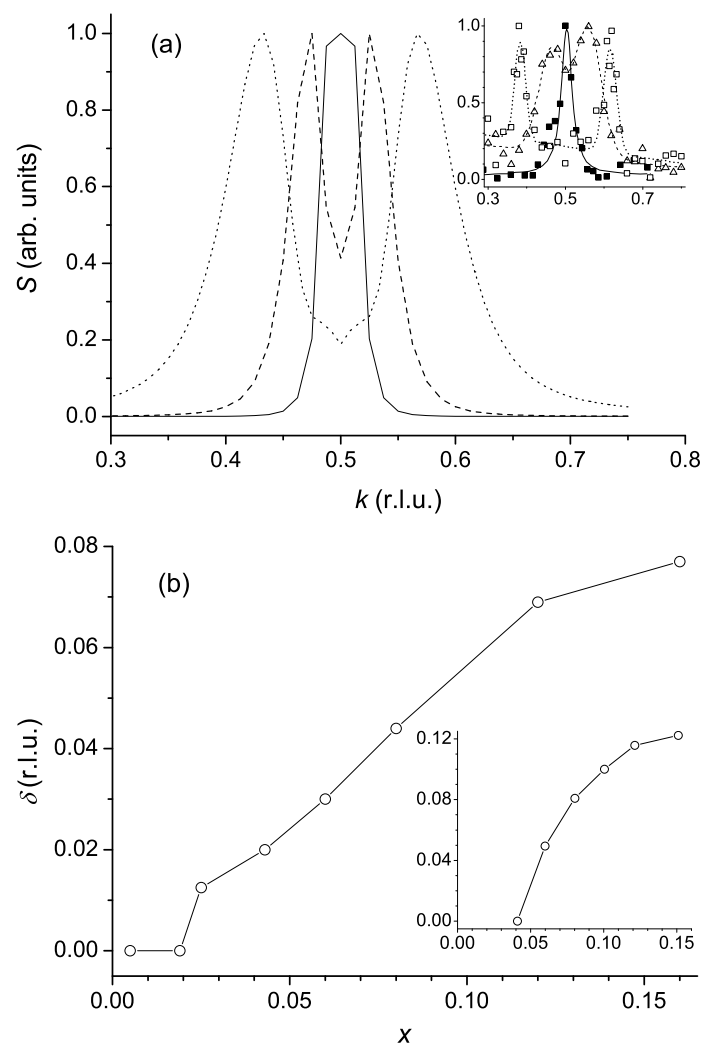

FIG. 3: (a) The dynamic structure factor vs. wave vector along the edge for $x=0.015$ (solid line), 0.06 (dashed line), and 0.12 (dotted line). For all curves $T=0.01 t$ and $\omega=0.004 t$. Inset: the structure factor measured [2, 21] in $\mathrm{La}_{2-x} \mathrm{Sr}_{x} \mathrm{CuO}_{4}$ for $x=0.03, T=2 \mathrm{~K}, \omega=0$ (filled squares and solid line), $x=0.06, T=25 \mathrm{~K}, \omega=2 \mathrm{meV}$ (triangles and dashed line), and $x=0.12, T=31 \mathrm{~K}, \omega=2 \mathrm{meV}$ (open squares and dotted line). Fitted curves in the inset are from 2, 21]. (b) The incommensurability parameter $\delta$ vs. $x$ for $T=0.01 t$ and $\omega=0.004 t$. Inset: experimental data 2] for $\mathrm{La}_{2-x} \mathrm{Sr}_{x} \mathrm{CuO}_{4}$. Connecting lines are a guide to the eye.

structure factor $S(\mathbf{k} \omega)$. We considered lattices up to $120 \times 120$ sites with the parameters $J=0.2 t$. Figure [2 demonstrates the dynamic structure factor calculated along edge and diagonal of the Brillouin zone near $\mathbf{Q}$. The parameters chosen are close to those used in experiments: for $t=0.5 \mathrm{eV}, T \approx 58 \mathrm{~K}$ and $\omega=2 \mathrm{meV}$. In agreement with experimental observations [1, 2, 3] peaks along the edge are more intensive than those along the diagonal.

Figure 3 shows the concentration dependence of the peak position along the edge. Starting from $x \approx 0.02$ there appears the incommensurability - instead of one peak at $\mathbf{Q}$ four peaks are observed at $\left(\frac{1}{2}, \frac{1}{2} \pm \delta\right)$ and $\left(\frac{1}{2} \pm \delta, \frac{1}{2}\right)$. For $x \lesssim 0.12$ the incommensurability parameter $\delta$ grows nearly linearly with growing $x$ and tends to saturation for $x>0.12$. As seen from the insets in Fig. B. this behavior is similar to the experimental observations except that in experiment the incommensurability arises 


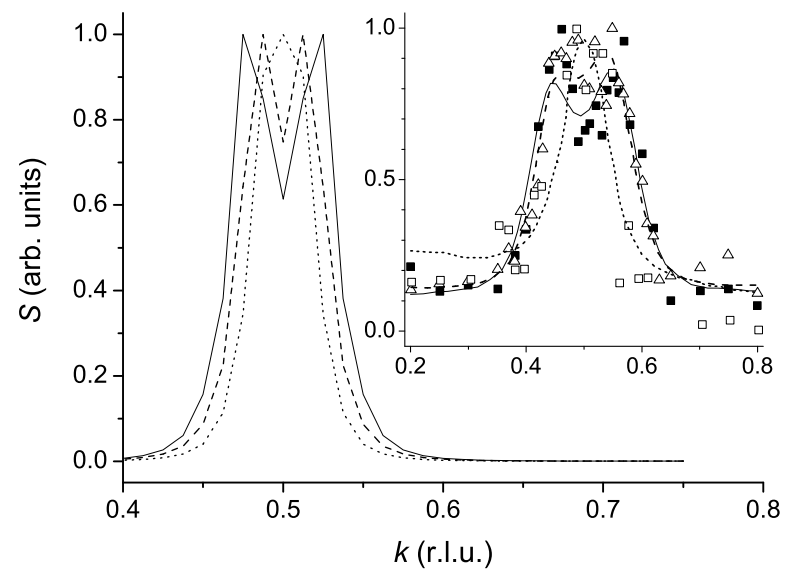

FIG. 4: The dynamic structure factor vs. wave vector along the edge for $x=0.04, \omega=0.004 t$ and $T=0.01 t$ (solid line), $0.02 t$ (dashed line), and $0.04 t$ (dotted line). Inset: the structure factor in $\mathrm{La}_{1.93} \mathrm{Sr}_{0.07} \mathrm{CuO}_{4}$ measured [22] for $\omega=4 \mathrm{meV}$, $T=23 \mathrm{~K}$ (filled squares and solid line), $T=100 \mathrm{~K}$ (triangles and dashed line), and for $\omega=8 \mathrm{meV}, T=293 \mathrm{~K}$ (open squares and dotted line). Fitted curves in the inset are from 22].

at $x \approx 0.04$ and the parameter $\delta$ is approximately 1.5 times larger. In our calculations its concentration dependence is mainly determined by the variation of the spin-gap parameter $\Delta$ in $\omega_{\mathbf{k}}$ in Eq. (4). For $x<0.12$ this parameter grows with $x$ and saturates at $x \approx 0.12[16$.

The temperature variation of the peaks is shown in

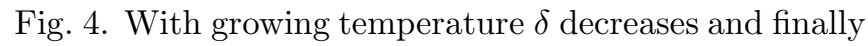
the incommensurability disappears. As seen in the inset of Fig. [4 the analogous behavior of the dynamic structure factor is observed experimentally [9, 22]. Notice that using Eq. (5) we neglected completely the damping in the hole spectral function. As mentioned, this damping decreases or even suppresses the incommensurability. The thermal growth of the damping hastens the decrease of the incommensurability with temperature.

It should be emphasized that at least in the case when magnons near $\mathbf{Q}$ are not overdamped the low-frequency incommensurability in $B(\mathbf{k} \omega)$ influences only weakly the hole spectral functions. The solution of the self-energy equations [16] for the $t$ - $J$ model indicates that in these conditions the density of holes remains homogeneous as it is apparently observed in the low-temperature orthorhombic phase in $\mathrm{La}_{2-x} \mathrm{Sr}_{x} \mathrm{CuO}_{4}$ [13].

In summary, it was shown that for hole concentrations $x \gtrsim 0.02$ the low-frequency magnetic response of the twodimensional $t-J$ model is incommensurate. The incommensurability is the consequence of the local minimum in the momentum dependence of the magnon damping. The minimum is located at the antiferromagnetic wave vector $\mathbf{Q}=\left(\frac{1}{2}, \frac{1}{2}\right)$ in the reciprocal lattice units and is connected with the growth of the phase space for the magnon damping into the electron-hole pair with dis- tance of the wave vector $\mathbf{k}$ from $\mathbf{Q}$. For such behavior the spin spectral function has to be strongly peaked near $\mathbf{Q}$ and the hole dispersion has to be approximately periodic with the period $\mathbf{Q}$. Both these conditions are fulfilled in crystals with short-range antiferromagnetic order to which lightly and moderately doped cuprates belong. In agreement with experiment for $x \lesssim 0.12$ the incommensurability grows nearly proportional to $x$ and tends to saturation for $x>0.12$. This dependence is connected with the concentration dependence of the spin gap at Q. Also in agreement with experiment the incommensurability decreases with increasing temperature. In the considered mechanism the magnetic incommensurability is not accompanied by the inhomogeneity of the carrier density.

This work was partially supported by the ESF grant No. 5548 and by DFG.

[1] H. Yoshizawa et al., J. Phys. Soc. Jpn. 57, 3686 (1988); R. J. Birgeneau et al., Phys. Rev. B 39, 2868 (1989).

[2] K. Yamada et al., Phys. Rev. B 57, 6165 (1998).

[3] T. E. Mason et al., Phys. Rev. Lett. 71, 919 (1993); M. Matsuda et al., Phys. Rev. B 49, 6958 (1994).

[4] T. Suzuki et al., Phys. Rev. B 57, R3229 (1998); H. Kimura et al., J. Phys. Soc. Jpn. 69, 851 (2000).

[5] N. Bulut et al., Phys. Rev. Lett. 64, 2723 (1990); Q. Si et al., Phys. Rev. B 47, 9055 (1993).

[6] B. Normand and P. A. Lee, Phys. Rev. B 51, 15519 (1995).

[7] V. Hizhnyakov and E. Sigmund, Physica C 156, 655 (1988); J. Zaanen and O. Gunnarson, Phys. Rev. B 40, 7391 (1989); S. R. White and D. J. Scalapino, Phys. Rev. Lett. 80, 1272 (1998).

[8] P. B. Littlewood et al., Phys. Rev. B 48, 487 (1993).

[9] M. Arai et al., Phys. Rev. Lett. 83, 608 (1999); P. Dai et al., Science 284, 1344 (1999).

[10] H. F. Fong et al., Phys. Rev. B 61, 14773 (2000).

[11] T. E. Mason et al., Phys. Rev. Lett. 77, 1604 (1996).

[12] M. Fujita et al., Phys. Rev. Lett. 88, 167008 (2002).

[13] H. Kimura et al., Phys. Rev. B 59, 6517 (1999).

[14] A. Sherman and M. Schreiber, Phys. Rev. B 68, 094519 (2003).

[15] Yu. A. Izyumov, Usp. Fiz. Nauk 167, 465 (1997) [Phys.Usp. (Russia) 40, 445 (1997)]; E. Dagotto, Rev. Mod. Phys. 66, 763 (1994).

[16] A. Sherman and M. Schreiber, Eur. Phys. J. B 32, 203 (2003); Phys. Rev. B 65, 134520 (2002).

[17] M. A. Kastner et al., Rev. Mod. Phys. 70, 897 (1998).

[18] D. K. Morr and D. Pines, Phys. Rev. Lett. 81, 1086 (1998).

[19] A. K. McMahan et al., Phys. Rev. B 42, 6268 (1990); V. A. Gavrichkov et al., Zh. Eksp. Teor. Fiz. 118, 422 (2000) [JETP (Russia) 91, 369 (2000)].

[20] A. Damascelli et al., Rev. Mod. Phys. 75, 473 (2003).

[21] S. Wakimoto et al., Phys. Rev. B 60, R769 (1999).

[22] H. Hiraka et al., J. Phys. Soc. Jpn. 70, 853 (2001). 